\title{
Postoperative neurological aggravation after anesthesia with sevoflurane in a patient with xeroderma pigmentosum: a case report
}

\author{
Salaheddine Fjouji, Mustapha Bensghir , Bahija Yafat, Najib Bouhabba, Elhoucine Boutayeb, Hicham Azendour
} and Nordine Drissi Kamili

\begin{abstract}
Introduction: Xeroderma pigmentosum is a rare autosomal recessive disease that causes changes in skin pigmentation, precancerous lesions and neurological abnormalities. It is a defect in the nucleotide excision repair mechanism. It has been reported that volatile anesthetics has a possible genotoxic side effect and deranged nucleotide excision repair in cells obtained from a patient with xeroderma pigmentosum.

We report an unusual case of postoperative neurological aggravation in a patient with xeroderma pigmentosum anesthetized with sevoflurane.

Case presentation: A 24-year-old African woman, who has had xeroderma pigmentosum since childhood, was admitted to our hospital for a femoral neck fracture. A preoperative physical examination revealed that she had a resting tremor with ataxia. She had cutaneous lesions such as keratosis and hyperpigmentation on her face and both hands. There was no major alteration of cognitive function, muscular strength was maintained and her osteotendinous reflexes were preserved. Surgical fixation was performed under general anesthesia after the failure of spinal anesthesia. All parameters were stable during surgery. When she woke up four hours later, the patient presented with confusion and psychomotor agitation, sharpened reflexes and the Babinski reflex was present. Her postoperative test results and a magnetic resonance imaging scan were unremarkable. It was suggested that sevoflurane had had a probable deleterious effect on the neurological status of this patient.
\end{abstract}

Conclusion: The anesthetizing of a patient with xeroderma pigmentosum is associated with a risk of worsening neurological disorders. At present, there are no clear recommendations to avoid the use of volatile agents in the anesthetic management of patients with xeroderma pigmentosum. More clinical and experimental research is needed to confirm the sensitivity of patients with xeroderma pigmentosum to sevoflurane and other halogenated anesthetics.

Keywords: Xeroderma pigmentosum, General anesthesia, Sevoflurane, Neurological worsening

\section{Introduction}

Xeroderma pigmentosum (XP) is a rare autosomal recessive disease, which is characterized by hypersensitivity of the skin to ultraviolet (UV) radiation and progressive neurological complications. It is caused by a defect in the nucleotide excision repair mechanism [1]. Estimated incidences vary from 1 in 20,000 in Japan to 1 in 250,000 in the USA, and approximately 2.3 per 1 million live births

\footnotetext{
* Correspondence: mustaphabens_15rea@hotmail.com

Department of Anaesthesiology, Military Hospital Med V Rabat, University of Med $\vee$ Souissi, Rabat, Morocco
}

(c) 2013 Fjouji et al.; licensee BioMed Central Ltd. This is an Open Access article distributed under the terms of the Creative

in Western Europe. Anecdotally, the incidence in North Africa and the Middle East is substantially higher [2]. Little information exists on the optimal anesthetic management of these patients [3-6]. It has been reported that volatile anesthetics have a genotoxic side effect in the cells of patients with XP and may worsen the symptoms $[5,7]$. However, up to now, there are no human data for this effect [8]. This report aimed at describing a postoperative neurological complication after general anesthesia in a patient with XP submitted to 
surgery for a femoral neck fracture and discussing the probability of sevoflurane as the factor of deterioration.

\section{Case presentation}

A 24-year-old African woman, who had had XP since childhood, was admitted to our hospital for a femoral neck fracture after a fall on the stairs. Surgical fixation was indicated. A preoperative evaluation could not determine the genetic type of her XP. She had minor cutaneous forms on her face and on her hands, and had no history of tumor surgery. A neurological examination showed a minor decline in intelligence quotient (IQ), and an end tremor of the extremities associated with ataxic gait. She was calm, cooperative and oriented in time and space, with preserved muscle strength and deep tendon reflexes with no anomalies. A preoperative magnetic resonance imaging (MRI) scan did not show any sign of brain abnormalities. Her cardiovascular and pulmonary evaluation and biological test results were normal. The patient was premedicated with oral hydroxizine $(50 \mathrm{mg}$ the day before and $50 \mathrm{mg}$ the morning of surgery). In the operating room, we initiated standard monitoring including pulse oximetry (SpO2), precordial cardioscopy, and noninvasive blood pressure (NIBP). A 16G peripheral venous catheter was secured and $1 \mathrm{~g}$ cefazolin was administered. The patient was placed in a sitting position for the spinal anesthesia, and an injection of hyperbaric bupivacaine $(12.5 \mathrm{mg})$ and fentanyl $(25 \mu \mathrm{g})$ was given using a $25 \mathrm{G}$ Tuohy needle after checking the flow of cerebrospinal fluid. However, the motor and sensory blocks were insufficient. The patient's Bromage score was 4 and her reaction to pinprick was significant. We converted to a general anesthesia induced by propofol $150 \mathrm{mg}$, fentanyl $250 \mu \mathrm{g}$, and cisatracurium $8 \mathrm{mg}$ and maintained by $2 \%$ sevoflurane with a $50 \%$ oxygen $50 \%$ air mixture. The procedure lasted 80 minutes without incident. The patient's NIBP reading was between 136 and 92 as maxima, 81 and $56 \mathrm{~mm} \mathrm{Hg}$ as minima, her heart rate was 85 to 105 beats/minute and her $\mathrm{SpO} 2$ was between 98 and $100 \%$. Postoperative analgesia was assured by paracetamol (1g), nefopam (20mg) and parecoxib (40mg). In the postoperative recovery room, the patient remained intubated and ventilated. Her unexplained nonawakening after about one hour induced us to monitor her postoperative residual curarization and to administer neostigmine. Her motor response was satisfactory. However, she did not wake up nor return to spontaneous ventilation after two hours, which induced us to perform a biological assessment (sodium, urea, glucose) that showed no abnormalities. After three hours, we noticed signs of awakening and spontaneous breathing. Her tidal volume was satisfactory. The patient became agitated, with abnormal movements that persisted despite the extubation, optimization of analgesia and a psychological reassurance. On examination, signs of pyramidal irritation appeared, such as sharpened reflexes, and the Babinski reflex was present, which necessitated transferring the patient to the MRI room for a scan; the images did not show any lesions that could explain these symptoms. Her agitation did reduce but other neurological disorders persisted for more than three postoperative days, with the appearance of memory disorders like false recognition and confusion in time and space. They were attributed to a neurological worsening of XP. Postoperative follow-up was recommended by neurologists. After three months, she is still presenting with confusion and cognitive decline, with memory disorders, ataxia and spasticity.

\section{Discussion}

$\mathrm{XP}$ is a rare autosomal recessive disease, which is characterized by hypersensitivity of the skin to ultraviolet (UV) radiation and progressive neurological complications. It is mainly characterized by dermatological manifestations such as atrophy, keratosis, telangiectasis, hyperpigmentation and neoplasias in areas exposed to sunlight. The ophthalmologic symptoms are photophobia, corneal lesions, bilateral cataracts and a higher risk for benign and malignant eye tumors. There are eight genetic types of XP [9]; seven different genes involved in classical XP (XPA to XPG) and one gene for XP variant. Only patients with XPC, XPE and XP variant do not have neurological disorders, while the others show major and progressive neurological abnormalities. In this report, the genetic type of XP was not known, but it was probably one of those associated with neurodegenerative symptoms. Neurological abnormalities are found in 18\% of XP patients [9]. The most common are mental retardation, spasticity, ataxia, microcephaly and peripheral neuropathy. Before the surgery, our patient had a minor decline of IQ, tremor of the extremities and ataxia. After surgery she appeared to develop irreversible cognitive decline, confusion, agitation and a pyramidal syndrome. Bone fragility in XP patients is probably due to a vitamin $\mathrm{D}$ deficiency related to patients' protection from sunlight.

Little information exists on the optimal anesthetic management of patients with XP. For this type of surgery, a spinal anesthesia was chosen initially to prevent any neurological repercussions that could be caused by general anesthesia. After the failure of this technique, the available alternative was a general anesthesia induced by propofol, fentanyl, and cisatracurium and maintained by sevoflurane. There was no event that could explain the irreversible postoperative neurological degradation in our case. There was no hemodynamic instability, no transient hypoxia, and no electrolytic disorder was found. In the recovery room, residual curarization was 
eliminated by monitoring and neostigmine administration. A postoperative MRI scan did not show any cerebral lesions. Neurological disorders occurred in our patient, which could be attributed to the worsening of her XP symptoms by peri-operative factors including anesthetic agents.

The contribution of general anesthesia/anesthetics to postoperative cognitive decline (POCD) as a clinical entity has not been established, because patients under general anesthesia do not have an increased rate of POCD compared with patients under regional anesthesia [8]. But, a recent pilot study indicates that patients under isoflurane anesthesia may have a higher rate of POCD than patients under desflurane anesthesia after lower extremity or abdominal surgery [10]. In this case, surgical factors appeared to be controlled, and neurotoxicity of the medication is probably the most likely etiology.

Beyond that, it is not possible to separate the inhalational anesthetic effects from those of other anesthetic agents. Some evidence suggests that these unwanted effects vary according to each hypnotic's specific pharmacodynamic and pharmacokinetic characteristics and its interaction with the individual patient [11]. Propofol reduces cerebral blood flow and cerebral $\mathrm{O} 2$ metabolism to a similar extent, has favorable pharmacokinetics and a high-quality recovery profile despite prolonged infusion $[12,13]$. Fentanyl is a half-life opioid analgesic. Its primary actions of therapeutic value are analgesia and sedation with a favorable side-effect profile and it may increase the patient's tolerance for pain $[14,15]$.

Data on volatile anesthetics-induced neurotoxicity in animal studies are accumulating rapidly [8]. However, there are, as yet, no human data for this effect. There has been no prospective randomized clinical trial to evaluate volatile anesthetics-induced neuroprotection or neurotoxicity [8]. For XP patients, Masuda et al. [5] recommend avoiding inhalation anesthetics and using total intravenous anesthesia (TIVA), based on a report by Reitz and Lanz [7]. These authors showed deoxyribonucleic acid (DNA) strand breaks and irreversible DNA exchange in the lymphocytes of two patients with XP after in vitro exposure to halothane and speculated about a possible genotoxic side effect of this drug. Karabiyik et al. [16] also showed transitory DNA damage by sevoflurane in human lymphocytes similar to isoflurane studied in vivo.

Associated with anesthetics-induced cell injury and death in neonatal animals, volatile anesthetics have also been shown to impair the cognitive functions of these animals [8]. There is, however, a lack of evidence for the causal relationship between the cell injury/death in the brain and the cognitive impairment in the animals after anesthetic exposure, both in the relationship between the genetic disorder in XP neuronal cells and the mechanism of sevoflurane-induced neurotoxicity.

On the other hand, volatile anesthetics have been used in clinical practice for nearly 160 years and are still the most commonly used anesthetics worldwide. Sevoflurane is often chosen for such patients based on its favorable properties for pediatric anesthesia, its ease of use, its malleability, and its clinical safety reported during its use even at high concentrations in inhalational anesthesia using a mask [4]. In our institution, sevoflurane has been used since 2006 with approximately 30 operations per day. Its safety is demonstrated but our experience with XP patients is still poor.

In clinical practice, among XP patients submitted to general anesthesia, Miyazaki et al. [6] reported a case of transient worsening of the neurological symptoms after anesthesia with volatile agents in previous surgery, whereas the intraoperative management and the postoperative course were uneventful with TIVA. Because the neurological deterioration in our patient appears to be probably related to the deleterious effects of anesthetics and sevoflurane is probably the most likely etiology because of its potential genotoxicity, we would suggest more experimental and clinical studies to affirm and explain a probable sensitivity of the neurodegenerative form of XP to volatile anesthetics. In the meantime, a preoperative neurological assessment is especially necessary in patients with the neurodegenerative genetic type $\mathrm{XP}$, and using the TIVA option is preferable.

\section{Conclusion}

The anesthesia of a patient with XP is associated with a risk of worsening neurological disorders. Because of their genotoxicity, based on experimental studies and case reports, the use of volatile anesthetics may have an adverse effect that has not yet been confirmed. At present, there are no clear recommendations to avoid volatile agents in the anesthetic management of patients with XP. More clinical and experimental research is needed to confirm the sensitivity of XP patients to sevoflurane and other halogenated anesthetics.

\section{Consent}

Written informed consent was obtained from the patient's next-of-kin for publication of this manuscript and accompanying images. A copy of the written consent is available for review by the Editor-in-Chief of this journal.

\section{Abbreviations}

MRI: Magnetic resonance imaging; NER: Nucleotide excision repair; NIBP: Noninvasive blood pressure; POCD: Postoperative cognitive decline; SpO2: Pulse oximetry; TIVA: Total intravenous anesthesia; UV: Ultraviolet; XP: Xeroderma pigmentosum.

\section{Competing interests}

The authors declare that they have no competing interests. 


\section{Authors' contributions}

$\mathrm{SF}, \mathrm{MB}, \mathrm{BY}$ and $\mathrm{NB}$ analyzed and interpreted the patient data. SF, MB and $\mathrm{EB}$ were major contributors in writing the manuscript. HA and NDK made the final corrections. All authors read and approved the final manuscript.

Received: 13 May 2012 Accepted: 14 February 2013

Published: 14 March 2013

\section{References}

1. Stefanini M, Kraemer KHK: Xeroderma pigmentosum. In Neurocutaneous Diseases. 51st edition. Edited by Ruggieri M, Pascual-Castroviejo I, Di Rocco C 2008:771-792.

2. Lehmann AR, McGibbon D, Stefanini M: Xeroderma pigmentosum. Orphanet J Rare Dis 2011, 6:70.

3. Brunner T, Jöhr M: Anesthetic management of a child with xeroderma pigmentosum. Paediatr Anaesth 2004, 14:697-698.

4. Oliveira CR, Elias L, Barros AC, Conceição DB: Anesthesia in patient with Xeroderma pigmentosum: case report. Rev Bras Anestesiol 2003, 53:46-51.

5. Masuda Y, Imaizumi H, Okanuma M, Narimatsu E, Asai Y, Namiki A: Anesthesia for a patient with xeroderma pigmentosum. Masui 2002, 51:169-171.

6. Miyazaki R, Nagata T, Kai T, Takahashi S: Anesthesia for a patient with xeroderma pigmentosum. Masui 2007, 56:439-441.

7. Reitz M, Lanz E: DNA strand breaks in cells with DNA repair deficiency after halothane exposure in vitro. Arzneimittelforschung 1993, 43:418-420

8. Zuo Z: Are volatile anesthetics neuroprotective or neurotoxic? Med Gas Res 2012, 2:10

9. Kraemer KH, Lee MM, Scotto J: Xeroderma pigmentosum. Cutaneous, ocular, and neurologic abnormalities in 830 published cases. Arch Dermatol 1987, 123:241-250.

10. Zhang B, Tian M, Zhen Y, Yue Y, Sherman J, Zheng H, Li S, Tanzi RE, Marcantonio ER, Xie Z: The effects of isoflurane and desflurane on cognitive function in humans. Anesth Analg 2012, 114:410-415.

11. Bilotta F, Doronzio A, Stazi E, Titi L, Zeppa IO, Cianchi A, Rosa G, Paoloni FP, Bergese S, Asouhidou I, loannou P, Abramowicz AE, Spinelli A, Delphin E, Ayrian E, Zelman V, Lumb P: Early postoperative cognitive dysfunction and postoperative delirium after anaesthesia with various hypnotics: study protocol for a randomised controlled trial-the PINOCCHIO trial. Trials 2011, 12:170

12. Lauta E, Abbinante C, Del Gaudio A, Aloj F, Fanelli M, de Vivo P, Tommasino C, Fiore $\mathrm{T}$ : Emergence times are similar with sevoflurane and total intravenous anesthesia: results of a multicenter RCT of patients scheduled for elective supratentorial craniotomy. J Neurosurg Anesthesiol 2010, 22:110-118.

13. Rasmussen M, Juul N, Christensen SM, Jónsdóttir KY, Gyldensted C, Vestergaard-Poulsen P, Cold GE, Østergaard L: Cerebral blood flow, blood volume, and mean transit time responses to propofol and indomethacin in peritumor and contralateral brain regions: perioperative perfusionweighted magnetic resonance imaging in patients with brain tumors. Anesthesiology 2010, 112:50-56.

14. Petersen KD, Landsfeldt U, Cold GE, Petersen CB, Mau S, Hauerberg J, Holst P, Olsen K: Intracranial pressure and cerebral hemodynamic in patients with cerebral tumors: a randomized prospective study of patients subjected to craniotomy in propofol-fentanyl, isoflurane-fentanyl, or sevofluranefentanyl anesthesia. Anesthesiology 2003, 98:329-336.

15. Trescot AM, Datta S, Lee M, Hansen H: Opioid pharmacology. Pain Physician 2008, 11:S133-S153.

16. Karabiyik L, Sardas S, Polat U, KocabaS NA, Karakaya AE: Comparison of genotoxicity of sevoflurane and isoflurane in human lymphocytes studied in vivo using the comet assay. Mutat Res 2001, 492:99-107.

doi:10.1186/1752-1947-7-73

Cite this article as: Fjouji et al:: Postoperative neurological aggravation after anesthesia with sevoflurane in a patient with xeroderma pigmentosum: a case report. Journal of Medical Case Reports 2013 7:73.

\section{Submit your next manuscript to BioMed Central and take full advantage of:}

- Convenient online submission

- Thorough peer review

- No space constraints or color figure charges

- Immediate publication on acceptance

- Inclusion in PubMed, CAS, Scopus and Google Scholar

- Research which is freely available for redistribution 